\title{
Intradialytic exercise in the treatment of social frailty during the COVID-19 pandemic: A single- center prospective study
}

Koki Abe ( $\nabla$ a.abe@sapmed.ac.jp )

Teine Keijinkai Medical Center

Yoshinosuke Shimamura

Teine Keijinkai Medical Center

Takuto Maeda

Teine Keijinkai Medical Center

\section{Yoshikazu Kato}

Teine Keijinkai Medical Center

Yasuyoshi Yoshimura

Teine Keijinkai Medical Center

\section{Tomomi Tanaka}

Teine Keijinkai Medical Center

Hideki Takizawa

Teine Keijinkai Medical Center

\section{Research Article}

Keywords: Hemodialysis, Intradialytic exercise, Social frailty, Physical function, COVID-19

Posted Date: June 23rd, 2020

DOI: https://doi.org/10.21203/rs.3.rs-37597/v1

License: (c) (i) This work is licensed under a Creative Commons Attribution 4.0 International License. Read Full License 


\section{Abstract}

Background: Social frailty-the lack of a connection to society and infrequent social activities-has been reported to be associated with future declines in physical function in elderly individuals. This study aimed to evaluate both the association of social frailty with the physical function and the efficacy of intradialytic exercise as a therapy for social frailty among hemodialysis patients.

Methods: A total of 16 hemodialysis patients in the hemodialysis department of a single medical center were enrolled in this single-center prospective single-arm interventional study. Patients received five questions which asked about going out infrequently, lack of visiting friends, feeling unhelpful to friends or family, living alone, and lack of talking with someone. Those to whom two or more of the above were applicable were categorized as socially frail. All patients were placed into exercise therapy to be performed during their thrice-weekly hemodialysis visits. Participants' physical function (walking speed), muscle strength (grip strength), muscle mass (appendicular skeletal muscle mass index), and social frailty were evaluated at baseline and after three months of therapy.

Results: Four (25\%) of the 16 participants (median age 71.5 years, 8 women) were categorized as being socially frail. In comparison to the non-socially frail group (non-SF), the socially frail group (SF) had a significantly lower walking speed $(0.70 \pm 0.12 \mathrm{~m} / \mathrm{s}$ vs $1.15 \pm 0.26 \mathrm{~m} / \mathrm{s}, p=0.005)$ and significantly worse performance on the Short Physical Performance Battery. Three months of intradialytic exercise therapy significantly improved their walking speed, from $1.04 \pm 0.30 \mathrm{~m} / \mathrm{s}$ to $1.16 \pm 0.29 \mathrm{~m} / \mathrm{s}(p=0.003)$. Intradialytic exercise therapy significantly improved walking speed in both the SF group and the non-SF group. The 2019 coronavirus disease pandemic occurred in the middle of the intervention period of this study, and although it was not statistically significant, the number of socially frail individuals among our participants increased to seven $(43.8 \%, p=0.248)$.

Conclusions: Social frailty was associated with reduced physical function among hemodialysis patients. Intradialytic exercise therapy improved physical function regardless of the presence of social frailty.

Trial registration: UMIN-CTR, UMIN-CTR000038313. Registered November 1, 2019,

https://upload.umin.ac.jp/cgi-open-bin/ctr_e/ctr_view.cgi?recptno=R000043639.

\section{Background}

Social frailty is the state of having reduced connections to society and little social activity. Among elderly community residents, social frailty has been reported to be significantly correlated with reduced physical function and future disability. ${ }^{1,2}$ As the coronavirus disease 2019 (COVID-19) pandemic continues to limit the social activity of people throughout the world, we must investigate how best to address social frailty.

Hemodialysis patients are also at high risk of losing physical function over time. ${ }^{3}$ However, the prevalence of social frailty among dialysis patients, the effects of social frailty on their physical function, 
and effective therapies of social frailty remain unclear.

This study aimed to evaluate both the association of social frailty with the physical function and the efficacy of intradialytic exercise as a therapy for social frailty among hemodialysis patients.

\section{Methods}

\section{Study Design and Intervention}

This was a single-center prospective single-arm interventional study. All 16 patients undergoing outpatient hemodialysis at our hemodialysis center as of November 1, 2019, were selected for enrollment into this study. Exclusion criteria were as follows: cases for which we were unable to obtain consent and cases with severe cardiac illnesses (acute myocardial infarction, unstable angina, arrhythmia with hemodynamic abnormalities, and advanced aortic stenosis). We obtained written, informed consent from all participants. None of the 16 selected participants met any of these exclusion criteria.

The intervention in this study was thrice-weekly intradialytic (i.e., during hemodialysis) exercise therapy. Patients underwent 50 minutes of exercise therapy during each of their three weekly hemodialysis visits. Exercise therapy began one hour after dialysis therapy had begun, and consisted of 10 minutes of stretching, 20 minutes of aerobic exercise using an ergometer at a rating of perceived exertion (RPE) of 13 ("somewhat hard"), and 20 minutes of resistance training using elastic bands and weights at a RPE of 13. Resistance training consisted of shoulder flexion/abduction, hip flexion/abduction, knee extension, and back bridge exercises, with 20 repetitions constituting one set of each exercise. Dedicated physical therapists were present to monitor exercise sessions and offer advice. In order to prevent the spread of COVID-19, dialysis staff, physical therapists, and patients were all subjected to daily morning measurements of body temperature, asked to wear masks, requested to follow cough etiquette, and to practice good handwashing. Staff that developed fever-like symptoms were instructed to stay at home until the possibility of COVID-19 infection could be ruled out. Patients that developed fever-like symptoms had their dialysis treatments adjusted so as not to occur alongside any other patients, and received dialysis therapy in a private room, and had their intradialytic exercise therapy postponed. ${ }^{4,5}$ This study was registered with the Japanese University Hospital Medical Information Network Clinical Trials Registry (UMIN 000038313). The study protocol used herein was approved by the Internal Review Board of the Teine Keijinkai Medical Center (IRB Approval No. 2-019071-00) and was carried out in accordance with the Declaration of Helsinki.

\section{Data Collection}

We recorded information on patients' demographic characteristics, history of dialysis, comorbidities, number of medications currently being taken, social frailty status, physical function, muscle strength, and muscle mass.

\section{Social Frailty}


In keeping with the approach taking by Makizako et al., we evaluated patients' social frailty status by asking five questions. ${ }^{1}$ These were as follows: (1) Do you go out less often now than you did a year ago? (2) Do you rarely visit the homes of your friends? (3) Do you feel that you are unhelpful to your family and friends? (4) Do you live alone? (5) Do you not have the opportunity to speak to someone every day? Patients that answered "Yes" to two or more of these questions were categorized as socially frail.

\section{Measurement of Outcome}

Patients were measured at baseline and after three months of therapy. Measurements of physical function were carried out on dialysis therapy days before dialysis. Outcomes of this study were improvements in patients' walking speed, physical function, muscle strength, muscle mass, and social frailty status after three months of treatment.

\section{Physical Function and Muscle Strength}

Physical function was evaluated via walking speed and the Short Physical Performance Battery (SPPB). Walking speed was measured as patients' normal walking speed, without speeding up or slowing down, over a distance of four meters. Speeds of $0.8 \mathrm{~m} / \mathrm{s}$ or lower were defined as reduced walking speed. ${ }^{3}$ The SPPB was carried out in accordance with the standard method: patients were given scores from 0-4 based on their performance in each of three tasks: balance test, gait speed test, chair stand test. These three scores were totaled to yield a composite score. ${ }^{6}$ Muscle strength was evaluated as grip strength, which was measured while the patient was standing with their arms extended. Left and right arms were each measured twice, and the highest value was recorded.

\section{Muscle Mass}

Muscle mass was evaluated using the appendicular skeletal muscle mass index (ASMI). First, patient muscle mass was recorded using bioelectrical impedance analysis (BIA), which was then divided by the patient's height in meters and squared to yield an ASMI value.

\section{Statistical Analysis}

Normal distribution was assessed by the Shapiro-Wilk test. Continuous variables are expressed as means and standard deviation (SD) or median and interquartile range (IQR: 25-75th percentile) depending on the results of the Shapiro-Wilk test. Categorical variables were expressed as frequency and percentage. Differences in continuous variables between patients with social frailty and those without were tested by Student's $t$-test or the Mann-Whitney $U$ test. Differences in continuous variables within a group were tested by paired $t$-test or the Wilcoxon signed-rank test. Differences in categorical variables between patients with social frailty and patients without were analyzed by Fisher's test. Differences in categorical variables within a group were tested by McNemar test. Spearman's rank correlation was used to determine the relationship between the gait speed and the social frailty score. A probability value of < 
0.05 was considered to be statistically significant. Statistical analysis was performed using EZR software (Jichi Medical University, Saitama, Japan).

\section{Results}

Patients' background characteristics by social frailty status are given in Table 1. Four (25\%) of the 16 participants in this study (median age 71.5 years, eight women) were deemed to be socially frail. In comparison to the non-socially frail group (non-SF), the SF group had a significantly slower walking speed $(0.70 \pm 0.12 \mathrm{~m} / \mathrm{s}$ vs $1.15 \pm 0.26 \mathrm{~m} / \mathrm{s}, p=0.005)$ and significantly worse performance on the SPPB. The number of socially frail responses on the SF questionnaire correlated negatively with walking speed $(r=-0.607, p=0.012$, Figure 1$)$ and the SPPB $(r=-0.653, p=0.006)$. Single regression analysis indicated that social frailty was a risk factor for reduced walking speed (odds ratio $33.0,95 \%$ confidence interval 1.56-698.00, $p=0.025)$.

Table 1 Baseline characteristics of study subjects

\begin{tabular}{lcccc} 
Characteristics & Total & Non-social Frailty & Social Frailty & \\
& $(\mathrm{n}=16)$ & $(\mathrm{n}=12)$ & $(\mathrm{n}=4)$ & P-value \\
\hline Age (years) & $71.5(64.8-78.0)$ & $70.5(64.8-73.5)$ & $76.0(69.5-78.3)$ & 0.43 \\
\hline Female sex (\%) & $8(50.0)$ & $6(50.0)$ & $2(50.0)$ & 1.0 \\
\hline Height (cm) & $159.6 \pm 10.4$ & $161.2 \pm 8.6$ & $154.6 \pm 14.9$ & 0.28 \\
\hline Body weight (kg) & $51.4 \pm 12.2$ & $52.1 \pm 12.7$ & $49.2 \pm 12.1$ & 0.69 \\
\hline Body mass index (kg/m $\left.{ }^{2}\right)$ & $19.5(18.5-20.7)$ & $19.1(17.8-20.7)$ & $20.3(20.0-20.7)$ & 0.47 \\
\hline Dialysis vintage (months) & $83.1 \pm 60.4$ & $68.4 \pm 49.0$ & $127.3 \pm 77.3$ & 0.092 \\
\hline Charlson comorbidity index & $5.0(4-6.3)$ & $5.0(4.8-6.3)$ & $4.0(4.0-4.8)$ & 0.23 \\
\hline Number of medications & $6.3 \pm 3.0$ & $6.2 \pm 3.4$ & $6.8 \pm 1.5$ & 0.75 \\
\hline Gait speed (m/sec) & $1.04 \pm 0.30$ & $1.15 \pm 0.26$ & $0.70 \pm 0.12$ & 0.005 \\
\hline SPPB & $12.0(10.0-12.0)$ & $12.0(12.0-12.0)$ & $10.0(9.5-10.3)$ & 0.008 \\
\hline Grip strength (kg) & $25.1 \pm 7.7$ & $25.8 \pm 6.9$ & $22.9 \pm 10.6$ & 0.53 \\
\hline ASMI (kg/m $\left.{ }^{2}\right)$ & $6.4 \pm 0.9$ & $6.6 \pm 0.9$ & $6.1 \pm 1.1$ & 0.46 \\
\hline & & & & \\
\hline
\end{tabular}

All measurements are represented as number $(\%)$, mean \pm standard deviation, or median (interquartile range). 
Abbreviations: SPPB, short physical performance battery; ASMI, Appendicular skeletal muscle mass.

The outcomes of physical function, muscle strength, and muscle mass are listed in Table 2. Three months of intradialytic exercise therapy improved walking speed from $1.04 \pm 0.30 \mathrm{~m} / \mathrm{s}$ to $1.16 \pm 0.29 \mathrm{~m} / \mathrm{s}$ $(p=0.003)$; however, no significant change in SPPB performance was observed. ASMI and grip strength likewise did not change. A significant improvement in walking speed was seen in both the SF and non-SF groups. Additionally, the improvement was larger in the SF group (Figure 2).

Table 2 Changes in physical function, muscle strength, muscle mass, and social frailty

\begin{tabular}{lccc} 
& Baseline & 3 months & P-value \\
\hline Total (n=16) & & & \\
\hline Gait speed (m/sec) & $1.04 \pm 0.30$ & $1.16 \pm 0.29$ & 0.003 \\
\hline SPPB & $12.0(10.0-12.0)$ & $12.0(12.0-12.0)$ & 0.17 \\
\hline Grip strength (kg) & $25.1 \pm 7.7$ & $24.5 \pm 7.4$ & 0.39 \\
\hline ASMI (kg/m $\left.{ }^{2}\right)$ & $6.4 \pm 0.9$ & $6.4 \pm 1.0$ & 0.64 \\
\hline The number of socially frail responses & $1.0(1.0-1.3)$ & $1.0(1.0-3.0)$ & 0.071 \\
\hline Non-social Frailty (n=12) & & & \\
\hline Gait speed (m/sec) & $1.15 \pm 0.26$ & $1.24 \pm 0.27$ & 0.028 \\
\hline Social Frailty (n=4) & & & \\
\hline Gait speed (m/sec) & $0.70 \pm 0.12$ & $0.91 \pm 0.20$ & 0.0497 \\
\hline
\end{tabular}

All measurements are represented as mean \pm standard deviation or median (interquartile range).

Abbreviations: SPPB, short physical performance battery; ASMI, appendicular skeletal muscle mass.

Changes in indices of social frailty are given in Table 3. The number of individuals deemed socially frail increased from four to seven. Despite the fact that this increase was not statistically significant, a trending increase in the number of socially frail responses to our questionnaire was observed $(p=0.07$, Table 2). During our research period, the question item whose responses showed the worst result was the frequency of going out.

Table 3 Changes in subitems of social frailty 
Total $(\mathrm{n}=16)$

\begin{tabular}{lll} 
& Baseline & 3 months \\
\hline Going out infrequently (\%) & $8(50)$ & $11(69)$ \\
\hline Lack of visiting friends (\%) & $5(31)$ & $6(38)$ \\
\hline Feeling unhelpful to friends or family (\%) & $5(31)$ & $5(31)$ \\
\hline Living alone (\%) & $4(25)$ & $5(31)$ \\
\hline Lack of talking with someone (\%) & $2(13)$ & $3(19)$ \\
\hline Social frailty (\%) & $4(25)$ & $7(44)$ \\
\hline
\end{tabular}

All measurements are represented as number (\%).

No complications arose as a result of the intradialytic exercise therapy performed in this study. Further, no patients developed COVID-19 during the interventional period of this study, and no patients needed to suspend their dialysis therapy regimen.

\section{Discussion}

\section{Summary of results}

In this study, we evaluated the association of social frailty with the physical function and the efficacy of intradialytic exercise therapy in hemodialysis patients. Our results showed that social frailty was significantly correlated with decreased physical function and intradialytic exercise therapy improved patients' walking speeds regardless of their social frailty status.

\section{Most novel finding}

We have demonstrated that social frailty was significantly correlated with decreased physical function among hemodialysis outpatients. The prevalence of social frailty observed in this study was higher than that reported among the general elderly population. A retrospective cohort study of elderly community residents reported a social frailty prevalence of $10.2 \% .{ }^{1}$ A prior study demonstrated that social frailty was associated with a loss of physical functionality and disability in community-dwelling elderly population. ${ }^{2}$ However, to our knowledge, this was the first study to show that social frailty was also correlated with decreased physical function in hemodialysis patients.

\section{Potential mechanism}

Our result can be explained by the fact that hemodialysis patients are prone to have sedentary lifestyles and to have low levels of physical activity. ${ }^{7,8}$ Other studies also reported that decreased physical activity was associated with reduced walking speed in hemodialysis patients. ${ }^{9,10}$ We can speculate that social 
frailty such as a reduced frequency of going out and social visits with friends, reduces walking speed by limiting patients' opportunities to engage in physical activity.

\section{Secondary finding}

We showed intradialytic exercise therapy contributed to improving patients' walking speeds both in the SF group and the non-SF group. Both intradialytic and interdialytic exercise have been shown to be effective. ${ }^{11}$ We selected intradialytic exercise because intradialytic cycling and resistance training with guidance were shown to be effective in a short period of time. ${ }^{12,13}$ In contrast, interdialytic exercise, including home exercise therapy, had poor adherence. ${ }^{14,15}$

\section{Implications of the study}

During our study, the first Japanese case of COVID-19 was reported on January 15. 2020. As of April 30, the Japanese government has intermittently requested the public to refrain from leaving their homes. ${ }^{16}$ The COVID-19 pandemic, which occurred in the middle of this study, has impacted on our patients because three new cases of social frailty arose. Thus, the number of dialysis patients with social frailty is expected to be increased during the COVID-19 pandemic, suggesting the need for care and monitoring to avoid further declines in physical function among this vulnerable population.

\section{Limitations}

This study was subject to several limitations. First, it was a single-center study, meaning that some bias in patient selection may be present. Second, because its sample size is small, the effect of social frailty on the physical function could not be adequately adjusted for confounding factors like age, comorbidities, nutritional status, and cognitive function. A multicenter, large-scale trial is therefore necessary. Third, our study period was relatively short, so that long-term outcomes were not evaluated.

\section{Conclusions}

We conducted a single-center interventional trial of intradialytic exercise therapy in hemodialysis patients. Our results suggest that social frailty is associated with reduced physical function and that, irrespective of social frailty status, intradialytic exercise therapy improved patients' physical function. Fortunately, none of our patients developed COVID-19 during the interventional period of this study, but it is crucial to investigate the effect of the COVID-19 pandemic on physical function and intradialytic exercise therapy in our patients in our future research.

\section{Abbreviations}

ASMI: appendicular skeletal muscle mass index

BIA: bioelectrical impedance analysis 
COVID-19: Coronavirus disease 2019

IQR: interquartile range

non-SF: non-social frailty

RPE: rating of perceived exertion

SD: standard deviation

SF: social frailty

SPPB: short physical performance battery

\section{Declarations}

\section{Ethics Approval and Consent to Participate}

The study protocol used herein was approved by the Internal Review Board of the Teine Keijinkai Medical Center (IRB Approval No. 2-019071-00) and was carried out in accordance with the Declaration of Helsinki. Informed consent was individually obtained from all participants included in the study.

\section{Consent for Publication}

All co-authors approved this submission. The patients consented to publish their information details.

\section{Availability of Data and Materials}

The datasets used and/or analyzed during the current study are available from the corresponding author on reasonable request.

\section{Competing Interests}

The authors declare that they have no competing interests.

\section{Funding}

This research did not receive any specific funding from grant agencies.

\section{Authors' Contributions}

KA designed the protocol, collected, analyzed, and interpreted the data, and drafted the manuscript. YS collected and interpreted the data, and critically revised the manuscript. HT designed the protocol, collected and interpreted the data, and critically revised the manuscript. TM, YK, YY, and TT contributed to the data collection. All authors read and approved the final manuscript. 


\section{Acknowledgments}

We would like to thank Editage (www.editage.jp) for English language editing.

\section{References}

1. Makizako H, Shimada H, Tsutsumimoto K, Lee S, Doi T, Nakakubo S, et al. Social frailty in community-dwelling older adults as a risk factor for disability. Journal of the American Medical Directors Association. 2015;16(11): 1003.e7-11.

2. Makizako H, Shimada H, Doi T, Tsutsumimoto K, Hotta R, Nakakubo S, et al. Social frailty leads to the development of physical frailty among physically non-frail adults: a four-year follow-up longitudinal cohort study. International Journal of Environmental Research and Public Health. 2018;15(3): 490.

3. Kittiskulnam P, Chertow GM, Carrero JJ, Delgado C, Kaysen GA, Johansen KL. Sarcopenia and its individual criteria are associated, in part, with mortality among patients on hemodialysis. Kidney International. 2017;92(1): 238-247.

4. Basile C, Combe C, Pizzarelli F, Covic A, Davenport A, Kanbay M, et al. Recommendations for the prevention, mitigation and containment of the emerging SARS-CoV-2 (COVID-19) pandemic in haemodialysis centres. Nephrology Dialysis Transplantation. 2020;35(5): 737-741.

5. Khan F, Amatya B. Medical rehabilitation in pandemics: towards a new perspective. Journal of Rehabilitation Medicine. 2020. Available from: doi: 10.2340/16501977-2676.

6. Guralnik JM, Simonsick EM, Ferrucci L, Glynn RJ, Berkman LF, Blazer DG, et al. A short physical performance battery assessing lower extremity function: association with self-reported disability and prediction of mortality and nursing home admission. Journal of Gerontology. 1994;49(2): M85-94.

7. Gomes EP, Reboredo MM, Carvalho EV, Teixeira DR, Carvalho LF, Filho GF, et al. Physical activity in hemodialysis patients measured by triaxial accelerometer. BioMed Research International. 2015. Available from: doi: 10.1155/2015/645645.

8. Wilkinson TJ, Clarke AL, Nixon DGD, Hull KL, Song Y, Burton JO, et al. Prevalence and correlates of physical activity across kidney disease stages: an observational multicenter study. Nephrology Dialysis Transplantation. 2019. Available from: doi: 10.1093/ndt/gfz235.

9. Kutsuna T, Matsunaga A, Matsumoto T, Ishii A, Yamamoto K, Hotta K, et al. Physical activity is necessary to prevent deterioration of the walking ability of patients undergoing maintenance hemodialysis. Therapeutic Apheresis Dialysis. 2010;14(2): 193-200.

10. Matsuzawa R, Matsunaga A, Wang G, Kutsuna T, Ishii A, Abe Y, et al. Habitual physical activity measured by accelerometer and survival in maintenance hemodialysis patients. Clinical Journal of the American Society of Nephrology. 2012;7(12): 2010-2016.

11. Fang HY, Burrows BT, King AC, Wilund KR. A comparison of intradialytic versus out-of-clinic exercise training programs for hemodialysis patients. Blood Purification. 2020;49(1-2): 151-157.

12. Groussard C, Rouchon-Isnard M, Coutard C, Romain F, Malarde L, Lemoine-Morel S, et al. Beneficial effects of an intradialytic cycling training program in patients with end-stage kidney disease. Applied 
Physiology, Nutrition, and Metabolism. 2015;40(6): 550-556.

13. Molsted S, Harrison AP, Eidemak I, Andersen JL. The effects of high-load strength training with protein- or nonprotein-containing nutritional supplementation in patients undergoing dialysis. Journal of Renal Nutrition. 2013;23(2): 132-140.

14. Manfredini F, Mallamaci F, D'Arrigo G, Baggetta R, Bolignano D, Torino C, et al. Exercise in patients on dialysis: a multicenter, randomized clinical trial. Clinical Journal of the American Society of Nephrology. 2017;28(4): 1259-1268.

15. Delgado C, Johansen KL. Barriers to exercise participation among dialysis patients. Nephrology Dialysis Transplantation. 2012;27(3): 1152-1157.

16. Furuse Y, Ko YK, Saito M, Shobugawa Y, Jindai K, Saito T, et al. Epidemiology of COVID-19 outbreak in Japan, January-March 2020. Japanese Journal of Infectious Diseases. 2020. Available from: doi: 10.7883/yoken.JJID.2020.271.

\section{Figures}




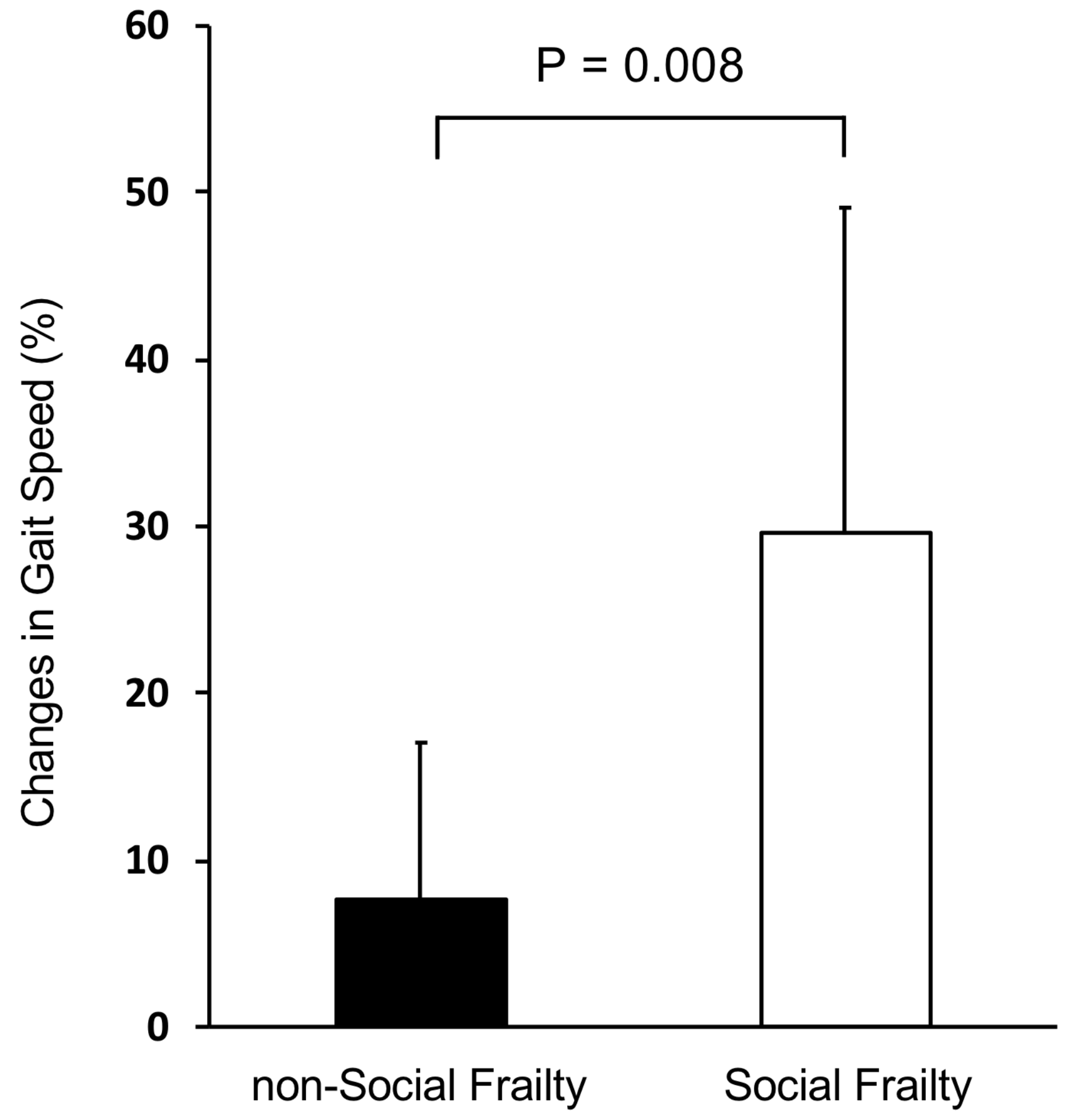

Figure 1

Percent changes in gait speed. Data are shown as mean and standard deviation. 


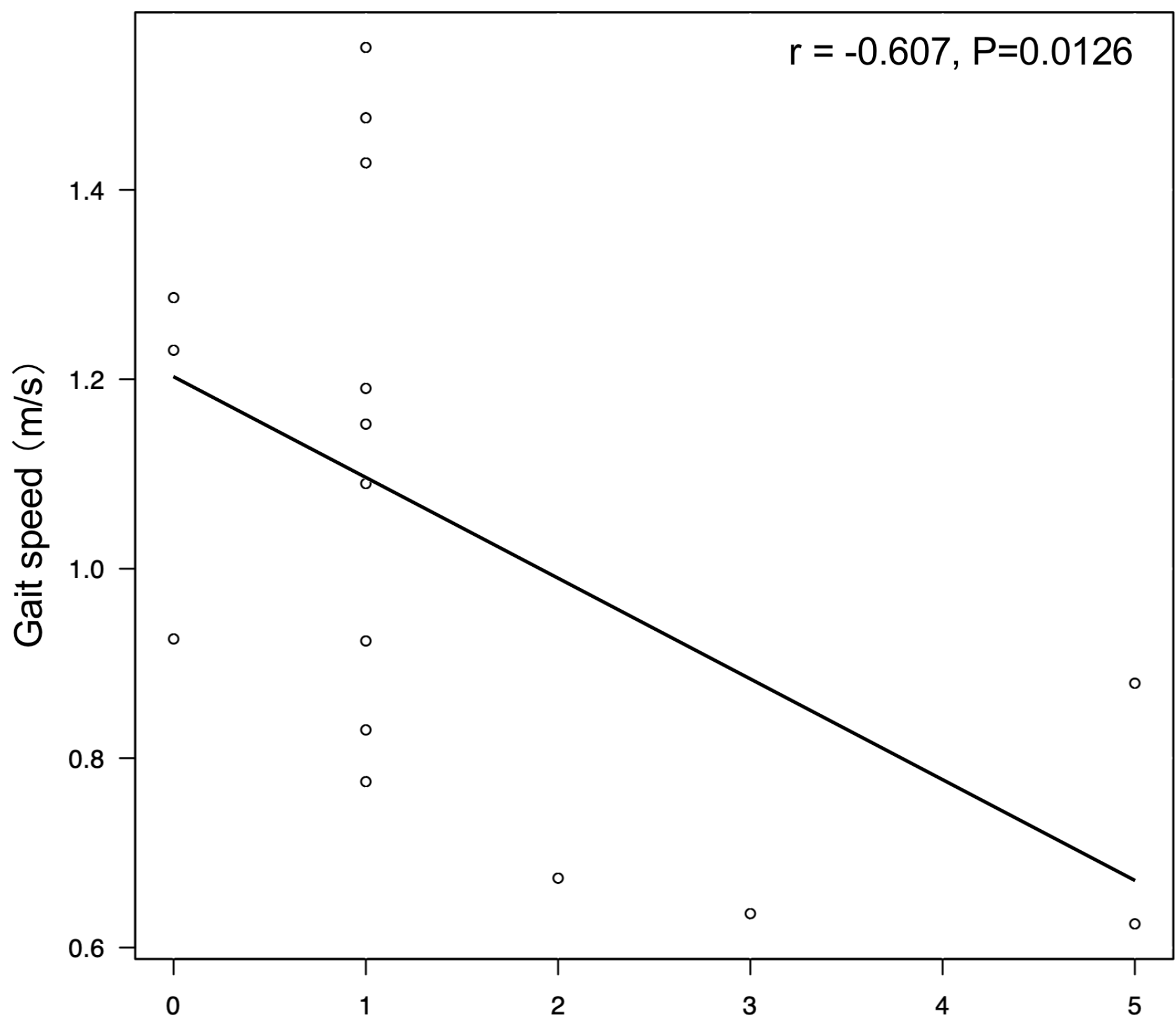

The number of socially frail responses on the five questionnaire

Figure 2

Correlation between social frailty score and gait speed. 